\author{
Military Technical College \\ Kobry Elkobbah, \\ Cairo, Egypt
}

$8^{\text {th }}$ International Conference on Aerospace Sciences \& Aviation Technology

\title{
THROUGHPUT ANALYSIS OF GLOBALSTAR SATELLITE SYSTEM
}

* Abd El-Aziz El-Basioni **Hebat Allah Murad "** Sherief Samy Emam

\section{ABSTRACT}

Throughput performance of Globalstar Low Earth Orbit (LEO) satellite system is considered. The effect of Multiple Access Interference (MAI) on throughput performance of a direct sequence spread spectrum LEO satellite communications network is discussed under non-uniform traffic model. Both Dense Traffic Satellite (DTS) and Sparse Traffic Satellite (STS) throughput are calculated. Finally we compare the normalized throughputs of both DTS and STS

\section{KEYWORDS}

Low Earth Orbit Satellite Communication.

\section{INTRODUCTION}

LEO satellite systems are now a reality. Iridium, Global star, Oddessy and

* Lucent Technologies, Cairo Office.

** Professor, Dept. of Communication, Faulty of Engineering, Cairo University, Giza, Egypt.

${ }^{* * *}$ M. Sc, Dept. of Communication Military technical college. 
teledesic are some LEO systems. Although mobile communication terrestrial system coverage is rapidly growing, they are limited to populated areas. Large areas of the Globe are rarely populated. LEO satellite systems are entitled for those rarely populated areas. So, LEO satellite systems are not competitor to mobile communication systems, but they are complementary to them. Iridium is a TDMA based system, while Globalstar and Oddessy are CDMA ones. The performance of any communication system is best illustrated by its throughput. The throughput of a communication system is defined as the average number of packets that are successfully delivered to receivers from the total number of transmitted packets. In this paper we consider the throughput analysis of the Globalstar system. We assume a flexible traffic distribution which cover both uniform and non-uniform traffic models. Throughput of both DTS and STS as well as the area covered by three consecutive satellites are calculated. The traffic model is given in section II. The throughput analysis is given in section III. The numerical results are given in section IV. We conclude the paper in section V.

\section{The Traffic Model}

Because of small coverage area of LEO Satellites, compared with geostationary ones, for a global communication network it is necessary to organize the LEO satellites on a multiple orbit configuration. In this model, an area on the earth is represented by an arc as shown in Fig. 1. In this figure, we distinguish between the coverage area of a satellite and the interference area of a satellite. The coverage area is specified by the minimum value of the elevation angle, $\theta_{\min }$ that an earth station is assumed to be able to access to the satellitfs while the interference area is determined by the final line of sight of that 


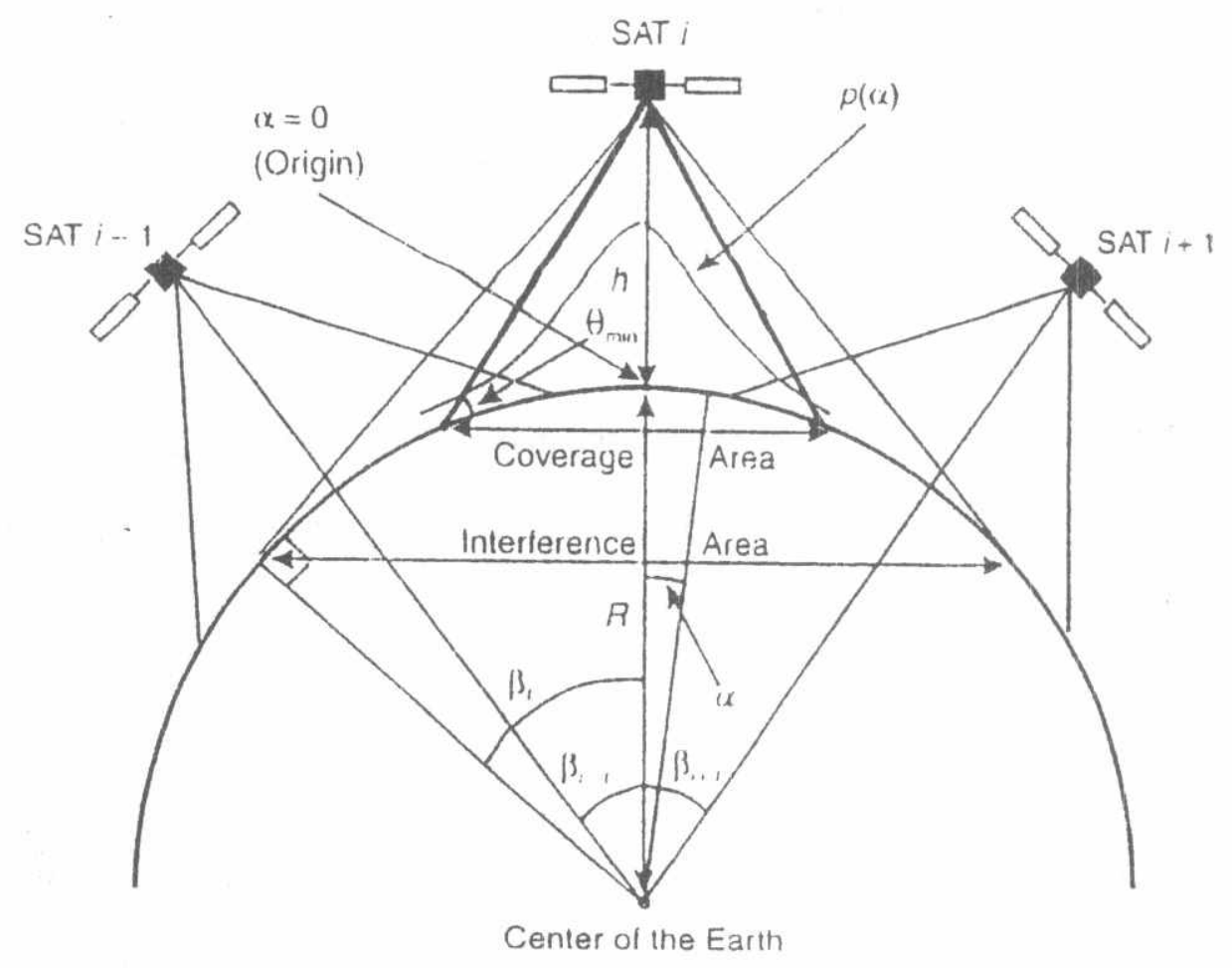

Fig.1. Typical shape of the normal non-uniform traffic model, $p(\alpha)$.

satellite. The service area is defined as a limited area within a coverage area where users can connect to satellite. The double coverage area is an area commonly located between two or more adjacent coverage areas. It should be noted that if an earth station lies in the interference area but out of the coverage area of a satellite, it would not be allowed to connect to that satellite, but still its signal reaches that satellite as interference. In order to analyze the influence of the non-uniformity in traffic, we focus on a series of three satellites and their users. Assume that a total numbers of users, N, are distributed randomly in a given area, the size of which is equal to the service areas of three adjacent satellites and their interference areas. For a LEO satellite system in which the satellites have the altitude $h(\mathrm{~km})$, the geometric interference limit for each of them in a smooth spherical earth assumption is given by the angle

$$
\beta_{I}=\cos ^{-1}\left(\frac{R}{R+h}\right)
$$


Were $R$ is the average radius of the earth. By this notation, we can say that $N$ users are distributed in an area whose size equals

$$
\frac{4 \pi}{N s}+2 \beta_{I}
$$

which is the size of the interference areas of the three succeeding satellites. In that area, it is assumed that the location of every user is a random variable with probability density function $p(\alpha)$ as [1]

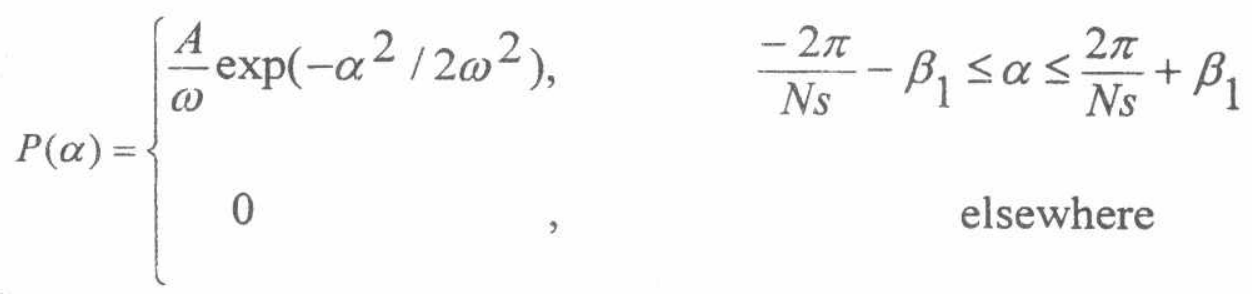

Where $\alpha$ is the relative location of a user, measured by the angle at the center of the earth; $\omega$ is the parameter representing uniformity in traffic; $N s$, is the number of satellites in each orbit; and $A$ is a factor that makes the total probability of the existence of a user in the area fixed and equal to 1 , when changing $\omega$ or $\mathrm{Ns}$, equal to

$$
A=\omega / \int_{-2 \frac{\pi}{\mathrm{Ns}}-\beta}^{2 \frac{\pi}{\mathrm{Ns}}+\beta} \exp \left(-\alpha^{2} / 2 \omega^{2}\right)
$$

Finally, it is assumed that each user sends a packet, including newly generated and retransmitted packets, with probability q [7]. If we assume $M$ users in the specific area under consideration, the distribution of the number of packets that are sent simultaneously to the channel becomes binomial with the parameters $q$ and $M$ as $[4]$ 


$$
f(m ; M)=\left\{\begin{array}{cc}
\left(\begin{array}{c}
M \\
m
\end{array}\right) \mathrm{q}^{\mathrm{m}}(1-\mathrm{q})^{\mathrm{M}-\mathrm{m}} & \mathrm{m} \leq \mathrm{M} \\
0 & \mathrm{~m}>\mathrm{M}
\end{array}\right.
$$

Where $\mathrm{m}$ is the number of transmitted packets. Here we consider the Globalstar LEO satellite system with eight orbits and six satellites on each orbit. For each satellite; say the ith satellite, let define three separate areas, as shown in Fig. 2.

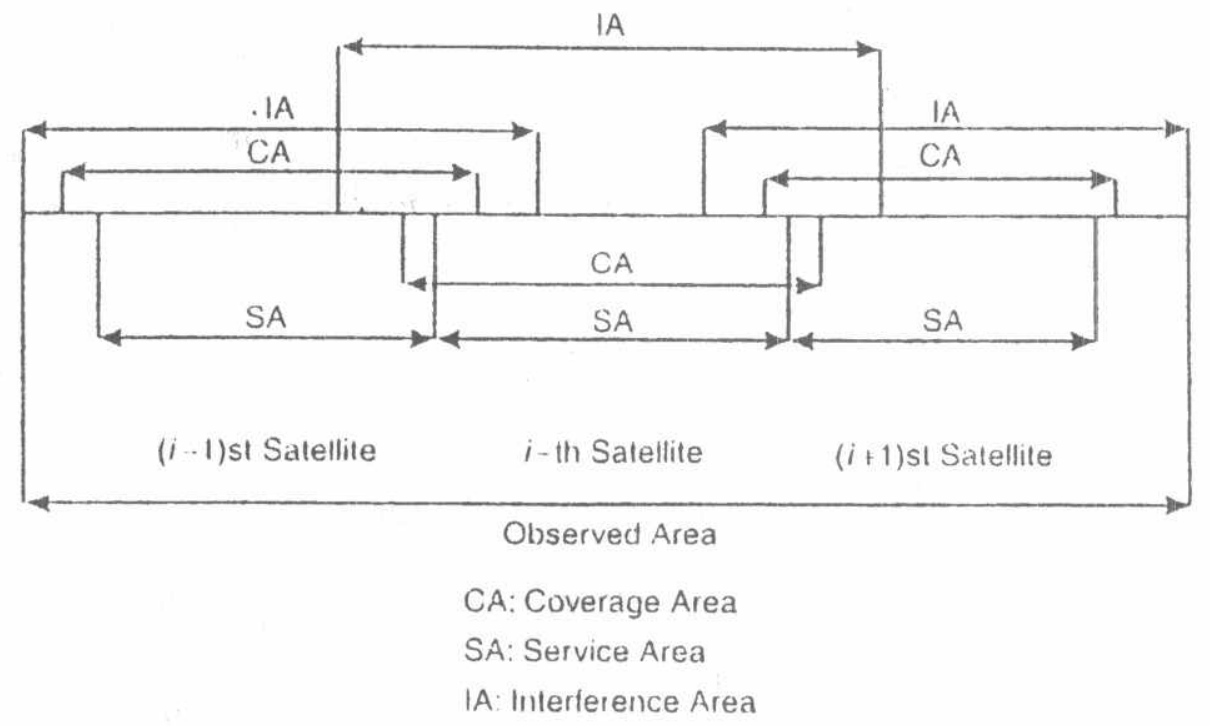

Fig.2. Definition of area 1, area 2, and area 3 for the ith satellite.

- area 1 : the intersection area between the service area of the $(i-1)$ th satellite and the visible area of the ith satellite;

- area 2 : the service area of the ith satellite;

- area 3 : the intersection area between the service area of the $(i+1)$ th satellite and the interference area of the ith satellite.

\section{Throughput Analysis}

In the case of LEO satellite systems with non-uniform traffic distribution, 
the expected number of users and hence the composite packet arrival rate are different in each service area. Thus, we normalize the throughput for each satellite by the expected number of users in its service area, $E\{\mathrm{Ni}\}$, namely, the normalized throughput for the ith satellite, as

$$
\xi_{i, \text { norm }}=\frac{\xi_{\mathrm{i}}}{\mathrm{E}\{\mathrm{Ni}\}} \quad, \mathrm{i}=1,2, \ldots \ldots, \mathrm{Ns}
$$

Where $\xi_{i}$ is the expected number of successfully transmitted packets of the ith sateliite. Assume that $c_{l}$ is the number of successful transmissions for the ith satellite among simultaneous $n_{i}$ packets transmitted from its service area when $m_{i}$ packets are sent from its interference area. In that case,

$$
p\left[c_{i}=c \backslash n_{i}=n, m_{i}=m\right]=\left(\begin{array}{l}
n \\
c
\end{array}\right) p_{c, i}^{c}(n, m)\left[1-p_{c, i}(n, m)\right]^{n-c}
$$

Where $P[A]$ define the occurrence probability of event $A$, and $P_{c, i}(n, m)$ is the probability of success for a packet in the presence of $n$ and $m$ packets in the service area and in the interference area of the ith satellite, respectively. The throughput for this case is the expected number of successful transmissions to the ith satellite, is given by

$$
\xi_{i}\left(n_{i}, m_{i}\right)=E\left[c_{i} \mid n_{i}, m_{i}\right]
$$

and it can be written in the form

$$
\xi_{\mathrm{i}}\left(\mathrm{n}_{\mathrm{i}}, \mathrm{m}_{\mathrm{i}}\right)=\sum_{\mathrm{c}=\mathrm{o}}^{n} \mathrm{c}\left({ }_{\mathrm{c}}^{\mathrm{n}}{ }_{\mathrm{i}} \mathrm{P}_{\mathrm{c}, \mathrm{i}}^{\mathrm{c}}\left(\mathrm{n}_{\mathrm{i}}, \mathrm{m}_{\mathrm{i}}\right)\left[1-\mathrm{P}_{\mathrm{c}, \mathrm{i}}\left(n_{i}, m_{i}\right)\right]^{\mathrm{n}_{\mathrm{i}}-\mathrm{c}}\right.
$$


The summation equals $n_{\mathrm{i}} . P_{c, i}(n, m)$, then,

$$
\xi_{i}\left(n_{i}, m_{i}\right)=n_{i} \cdot P_{c, i}\left(n_{i}, m_{i}\right)
$$

to find the total throughput of the ith satellite, we should average for all possible values of $n_{i}$ and $m_{i}$. To calculate that average value, we also should consider the probability of $n_{i}$ and $m_{i}$ packets from a total $N$ users. Hence, the throughput of the ith satellite, $\xi_{i}$ is the expected value of $\xi_{i}\left(n_{i}, m_{i}\right)$, that is,

$$
\begin{aligned}
& \xi_{\mathrm{i}}=\mathrm{E}\left\{\left(\mathrm{n}_{\mathrm{i}}, \mathrm{m}_{\mathrm{i}}\right)\right\}= \\
& \sum_{M=1}^{N} P\left[M_{i}=M\right] \sum_{m=1}^{i N} \mathrm{f}(\mathrm{m}, \mathrm{M}) \sum_{n=1}^{\mathrm{m}} \mathrm{P}[\mathrm{ni}=\mathrm{n} \backslash \mathrm{mi}=\mathrm{m}] \mathrm{nP} \mathrm{c}, \mathrm{i}_{\mathrm{n}}(n, m)
\end{aligned}
$$

Where $M_{i}$ is the number of users in the interference area, and $P\left[M_{i}=M\right]$, is the probability of $\mathrm{M}$ users in the interference area of the ith satellite, that is,

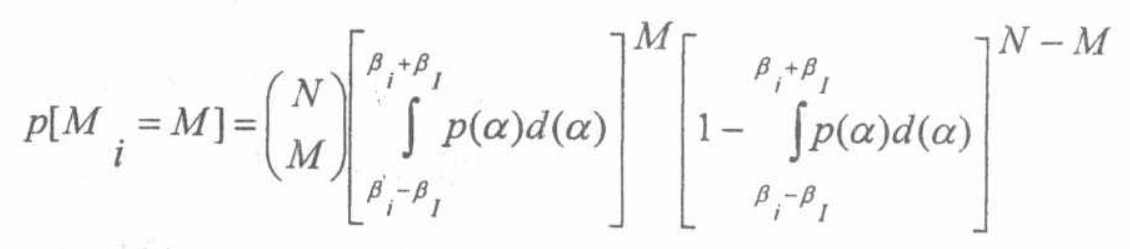

where $\beta_{i}$ is the angle between the ith satellite and the peak of the probability density function $P(\alpha)$. The conditional probability of $n$ packets in the service area of the ith satellite when there is information on existing $\mathrm{m}$ packets in the interference area of that satellite is equal to,

$$
P\left[n_{i}=n \mid \mathrm{m}_{\mathrm{i}}=\mathrm{m}\right]=\left(\begin{array}{l}
m \\
n
\end{array}\right) \varepsilon^{\mathrm{n}}(1-\varepsilon)^{\mathrm{m}-\mathrm{n}}
$$

where $\varepsilon$ is the probability of a single user in the service area when we know that 
the user is in the interference area of the same satellite and equals

$$
\varepsilon=P\left[n_{i}=1 \mid \mathrm{m}_{\mathrm{i}}=1\right]=\frac{\beta_{i}+\frac{\pi}{\mathrm{Ns}} P(\alpha) d \alpha}{\beta_{i}+\beta_{1}}
$$

And $P_{c, i}(n, m)$ is the probability of success [6].

To calculate the probability of packet success for the ones transmitting in the equivalent service area, we assume that the network topology is constant during each slot; hence, the level of interference is constant over a packet transmission. The summation of MAI and back ground Gaussian noise with two-sided power spectral density $N_{0} / 2$ is assumed as Gaussian noise. We can then define the equivalent bit energy-to-noise ratio at the ith satellite, $\mu_{\mathrm{i}}$. Assuming direct sequence modulation of the packets with binary phase shift keying (DS/BPSK), and rectangular chip pulse, we have [2]

$$
\mu_{i}=\left(\frac{2 I}{3 L S_{i}}+\frac{1}{\mu_{o}}\right)^{-1}
$$

Where $\mu_{0}$ is the ratio of bit energy to power spectral density of the background noise, $L$ is the bandwidth expansion factor, $S_{i}$ is the power of the received signal at the ith sate, ilite and $I_{i}$ is the total power of interference from $m$ users at ith satellite. $\mu$, also can be translated as the level signal-to-noise ratio (SNR) at each 
satellite in the absence of interferes. By modeling the total interference over a packet as Gaussian noise, it can be shown that the probability of packet success is a smooth function of signal -to-interference ratio, defined as the conditional probability of packet success as

$$
P\left[\text { success of tagged packet } / \mu_{\mathrm{i}}\right]=\mathrm{s}\left(\mu_{\mathrm{i}}\right)
$$

Since from packet to packet $\mu_{\mathrm{i}}$ is a random variable with probability density function $f_{\mu i}(\mu)$, the unconditional packet success probability, for a packet that is transmitted in the service area of the ith satellite will be given by

$$
p_{c, i}(m)=\int_{0}^{\infty} s(\mu) f_{\mu i}(\mu) d \mu
$$

Let

$$
K(\mu)=\frac{3 L}{2}\left(\frac{1}{\mu}-\frac{1}{\mu_{o}}\right)
$$

$K(\mu)$ denotes the ratio of power of interference to power of the desired signal at each satellite.

Abass [3] shows that $K(\mu)+1$ equals to the number of simultaneous transmissions to each satellite evaluated at a threshold level $\mu_{\mathrm{c}}$ for the SNR. He also shows that

$$
P_{c, i}(n, m)=F_{1}\left(K\left(\mu_{c}\right) S_{i}\right)
$$

For any given $(\mu)$ the probability of symbol error is given by[4]

$$
p_{e}=\frac{1}{2} \operatorname{erfc}(\sqrt{\mu})
$$


With the assumption of independent symbol errors, the conditional probability of packet success conditioned on SNR is given by

$$
\left.s(\mu)=\sum_{k=0}^{t}\left(\begin{array}{l}
n \\
k
\end{array}\right)\left(\frac{1}{2} \operatorname{erfd}(\sqrt{\mu})\right)^{k}\left(1-\frac{1}{2} \operatorname{erfd} \sqrt{\mu}\right)\right)^{n-k}
$$

Where $s(\mu)$ depends on t-error-correcting, and on the powerful error correcting method, it is close to a step function at some threshold value of SNR at the satellite, such as $\mu_{\mathrm{c}}$. This model is referred to as the threshold model, which is often used in the case of spread spectrum systems, and it means that the transmission of a packet is successful if the level of SNR is greater than the threshold. With this model the function $s(\mu)$ is

$$
s(\mu)=\left\{\begin{array}{lc}
1, & \mu \geq \mu_{c} \\
0, & \mu<\mu_{c}
\end{array}\right.
$$

By calculating the value of $\mathrm{K}(\mu)$ given in (16), at $\mu_{c}$, that is, $\mathrm{K}\left(\mu_{\mathrm{c}}\right)$, we can obtain the possible number of simultaneous transmissions, except for the tagged packet, in the absence of background noise and interference. Having the probabilities of packet success at the satellite(s) covering the equivalent service area, now we can calculate the throughput. If the expected number of users in the equivalent service area rand the total throughputs of the satellites covering this area (relating to the users in this area) are denoted by $E\{N\}$ and $\xi$, respectively, we normalize $\xi$ by $E\{N\}$ to obtain the normalized throughput, defined as

$$
\zeta_{\text {norm }}=\frac{\zeta}{E\{N\}} \quad(\text { packet / slot / user })
$$




\section{Numerical Results}

In this section we evaluate the normalized throughput of the Globalstar system for two adjacent satellites at the instant where one of the two satellites, say, the ith satellite, is just above the peak of the traffic load is the DTS, and its two adjacent neighbor satellites on both sides are the STS. The parameters of the Globalstar system are as follows: the number of orbits equals $8, N s=6, h=1400$ $\mathrm{km}, \theta_{\min }=10^{\circ}, B_{1}=35^{\circ}$, the coverage area of the ith satellite: $-26^{\circ} \leqslant \alpha \leqslant 26^{\circ}$, interference from $(i-1)$ th satellite: $-35^{\circ} \leqslant \alpha 1 \leqslant-34^{\circ}$, interference from $(i+1)$ th satellite: $34^{\circ} \leqslant \alpha 2 \leqslant 35^{\circ}$. The total number of users is assumed to be 100 , distributed in the area

$$
\frac{4 \pi}{N s}+2 \beta_{1}
$$

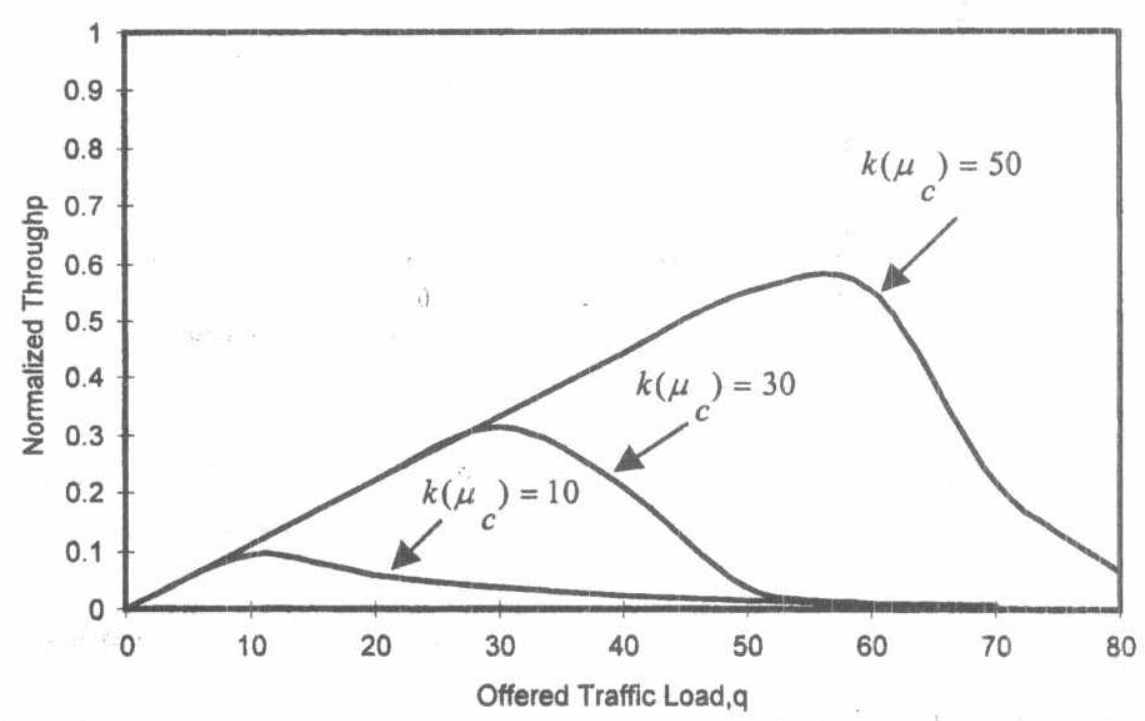

Fig.3. Normalized throughput as a function of total traffic for DTS

Fig.3. shows the normalized throughput performance as a function of total offered traffic load, qN for DTS. This figure is for a relatively non- uniform traffic 
Fig.3. shows the normalized throughput performance as a function of total offered traffic load, $\mathrm{qN}$ for DTS. This figure is for a relatively non- uniform traffic case, $\omega=0.5$, in which the average number of users of the DTS is about 64 . The performance of the DTS that should serve the major portion of users. As can be seen in the figure, with large multiple access capability as $k\left(\mu_{c}\right)=50$, in large offered traffic load's the throughput performance of the DTS still decreases. That also can be whien $K\left(\mu_{c}\right)=30$, even though the expected number of the users is much smaller 'chan 30 .

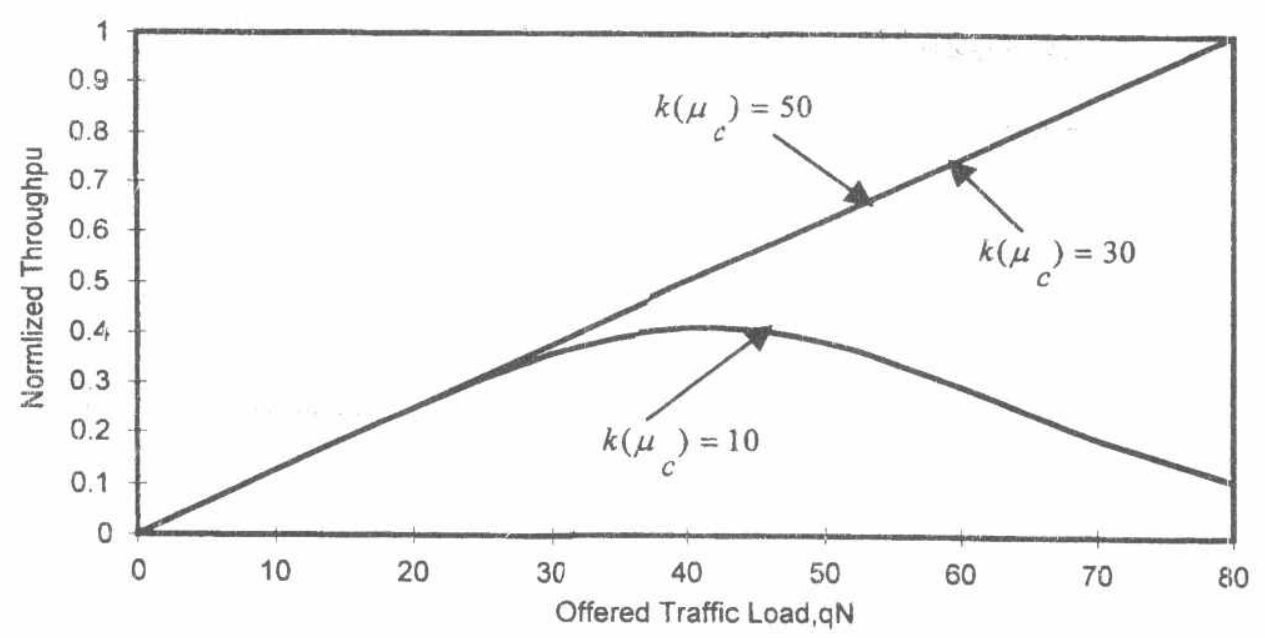

Fig.4. Normalized throughput as a function of total traffic for STS

Fig.4. Shows the normalized throughput performance as a function of total offered traffic load qN for STS. This figure is for a relatively non-uniform traffic case, $\omega=0.5$, in which the average number of users of the STS is about 12.

Figure 5 shows the normalized throughput performances as a function of total offered traffic load, q. N ior DTS and STS. From the figure, two facts can be pointed out: The first observation is the large difference in the performances of the DTS and the STS in the presence of non-uniform traffic. With the same value 
of $K\left(\mu_{c}\right)$, the performance of a few of the users of the STS is much better than the one for the number of the users of the DTS. The second point derived from the

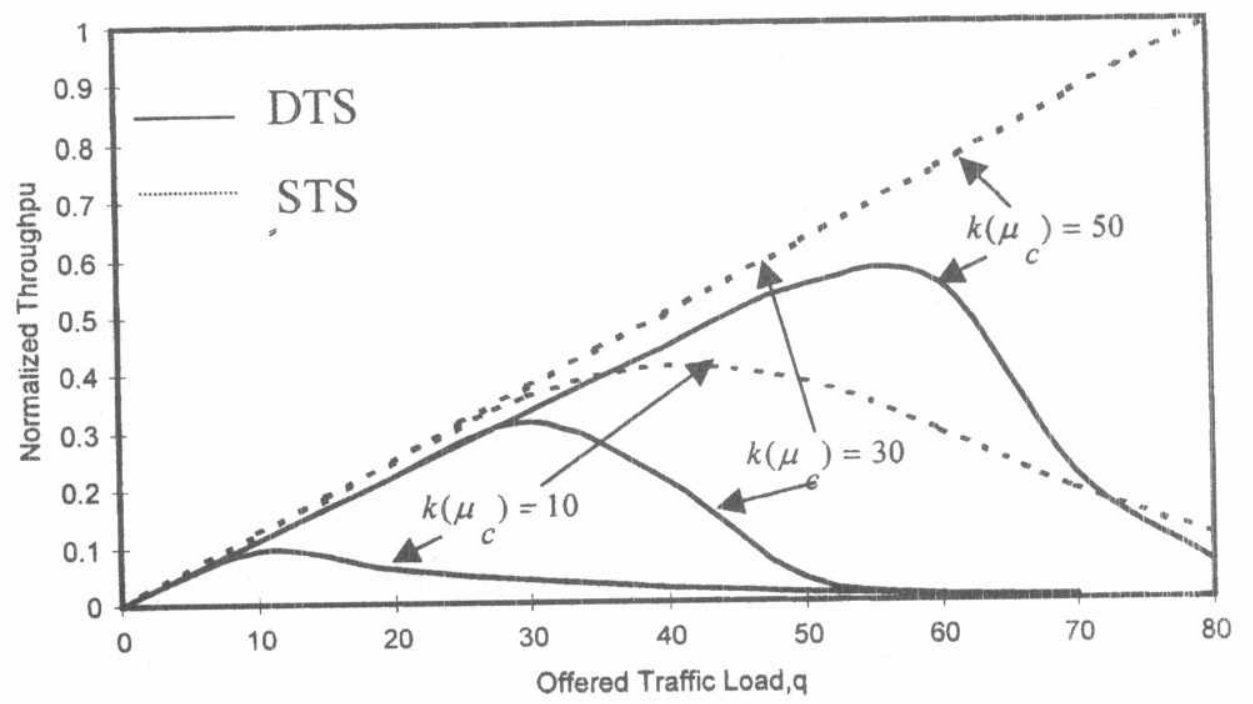

Fig.5. Normalized throughput as a function of total traffic for DTS and STS

figure is the performance of the DTS, that should serve the major portion of users. As can be seen in the figure, with a access capability as $k\left(\mu_{c}\right)=50$, in large offered traffic loads the throughput performance or the DTS stil decrease. That also can be when $\mathrm{K}\left(\mu_{\mathrm{c}}\right)=30$, even though t'ie expected number of the users is much smaller than 30 . Because of large interference power reached from the users of NTS. the performance of STS degrades in large offered traffic loads. I herefore, in LEO satellite comınunication systems, because of the existence of a large interference area compared to service area, the multiple access capability needs comparably large value than the expected number of the users.

\section{Conclusions}

In this paper, we have evaluated the throughput characteristics of a Global LEO satellite communications network. We assumed a non-uniform traffic 
and STS. It is shown that the throughput of the STS is better throughput of DTS where the MAI is larger. This suggests that some of the users under DTS could be transferred to STS. Another suggestion is to lower $\theta_{\min }$ in areas of lower population.

\section{References}

[1] A. Jamalipour et al., "performance of an integrated voice/Data system in non-uniform traffic low earth orbit satellite communication systems," IEEEJ. Select. Areas Commun., Vui. 13, No. 2, PP. 465-473, 1995.

[2] A. Jamalipour et al., "Transmit permission control on spread Aloha packets in LEO satellite systems," IEEE J. Select. Areas Commun., vol.14, pp. 1748757, Dec. 1996.

[3] A. Jamalipour, and A. Ogawa, "Packet Admission Control in a Direct Sequence Spread-Spectrum LEO Satellite Commun. Network, “IEEE J. Select. Areas

Commun., Vol. 15, No. 8, rP. 1649-1656, 1997.

[4] Kleinrock, L., Queuing systems, vols. 1 and 2, New York: Wiley, 1975.

[5] Proakis, J., Digitial C;ommunications, $2^{\text {nd }}$ ed., New York: McGraw-Hill, 1989.

[6] Pursley, M. B., "Performance Evaluation for Phase-Coded-Spread-Spectrum Multiple Access Communication Part 1: System Analysis," IEEE Trans. Commun., Vol. COM-25, No. 8, 1977, pp.795-799.

[7] Rayc'ıaudhuri, D., "Performance Analysis of Random Access PacketSv,vitched Code Division Multiple-Access Systems;" IEEE Trans. Commun., Vol. COM-29, No. 6, 1981, pp. 895-901. 